\title{
Monitoring offshore wind farm power performance with SCADA data and an advanced wake model
}

\author{
Niko Mittelmeier ${ }^{1}$, Tomas Blodau ${ }^{1}$, and Martin Kühn ${ }^{2}$ \\ ${ }^{1}$ Senvion GmbH, Überseering 10, 22297 Hamburg, Germany \\ ${ }^{2}$ ForWind - Carl von Ossietzky University of Oldenburg, Institute of Physics, Küpkersweg 70, \\ 26129 Oldenburg Germany \\ Correspondence to: Niko Mittelmeier (niko.mittelmeier@ senvion.com) \\ Received: 1 May 2016 - Discussion started: 17 May 2016 \\ Revised: 30 January 2017 - Accepted: 5 March 2017 - Published: 28 March 2017
}

\begin{abstract}
Wind farm underperformance can lead to significant losses in revenues. The efficient detection of wind turbines operating below their expected power output and immediate corrections help maximize asset value. The method, presented in this paper, estimates the environmental conditions from turbine states and uses precalculated lookup tables from a numeric wake model to predict the expected power output. Deviations between the expected and the measured power output ratio between two turbines are an indication of underperformance. The confidence of detected underperformance is estimated by a detailed analysis of the uncertainties of the method. Power normalization with reference turbines and averaging several measures performed by devices of the same type can reduce uncertainties for estimating the expected power. A demonstration of the method's ability to detect underperformance in the form of degradation and curtailment is given. An underperformance of $8 \%$ could be detected in a triple-wake condition.
\end{abstract}

\section{Introduction}

To increase the confidence in offshore wind energy investments, investors need reliable wind turbines. The two pillars of system reliability are operational availability and the ability to achieve predicted power performance. In the wind industry, the common standard IEC TS 61400-26-1 (2011) defines different categories of turbine conditions and describes the calculation of availability. However, within this standard the "full performance" category requires only a turbine status signal which confirms power production without any restrictions, but there is no verification of the quality of the power performance.

The key to an economic investment is a function of quantity and quality. Quantity is linked to availability and wind turbines can provide lots of SCADA (supervisory control and data acquisition) information which enables the analysis of time-based (IEC TS 61400-26-1, 2011) and productionbased availability (IEC TS 61400-26-2, 2014).

The quality of the power performance of a single turbine in specific conditions using a hub height met mast can be tested in accordance with the international standard IEC 61400-121 (2005). For most turbines in a typical wind farm, verification of the performance by comparison with the power curve is not suitable due to wake effects. Moreover, the installation and maintenance of a met mast is very expensive particularly offshore. Quantifying changes in power production based on wind speed measurements from nacelle anemometry relies on the quality of the device itself and its transfer function which should account for the flow distortion behind the rotor. This approach still requires us to find a wake-free sector and can lead to an increase in uncertainties (Albers et al., 1999; IEC 61400-12-2, 2013).

The efficient detection of underperformance of wind turbines increases asset value (Albers, 2004a). Incorrect turbine parameter settings, degradation of the blades, and pitch or yaw errors all lead to less production than expected. We differentiate between degradation and curtailments. A curtailed turbine has a limited power output below its expected power. Possible reasons for curtailments are load or sound reductions or grid requirements. For these incidents, turbine pa- 
rameters are changed on purpose and therefore documented in the turbine's SCADA logs. A turbine that is degraded reaches rated power but does not fulfil its expected power curve. These kinds of underperformance are more difficult to detect, especially when operating in the wake of neighboring wind turbines.

Albers (2004b) has published two methodologies for wind turbine performance evaluation. His integral model uses available wind conditions from the energy production of neighboring wind turbines (WTs), met masts or a combination of both and transfers the information via flow modeling and wake modeling to the investigated wind farm. The measured yield is corrected for turbine availability and then compared against the modeled yield in absolute values. Due to high uncertainties in flow and wake modeling this method is only proposed as a first general check. To reveal smaller deviations he proposes a relative wind turbine performance evaluation model. For this method, the active power values of direct neighbors are plotted against each other, and by comparing two periods, changes can be evaluated. This method explicitly excludes the sectors where wakes affect one or both turbines.

An international working group (IEC TC88 WG6, 2005) tried to come up with a standard for wind farm power performance testing. The proposed method uses one or more met masts to establish a measured wind farm power curve matrix. This two-dimensional measured power matrix (wind direction, wind speed) is compared against a modeled power matrix taking wake effects into account (Mellinghoff, 2006; Carvalho and Guedes, 2009). The standard could not be established.

Mittelmeier et al. (2013) presented a new method that uses relations between an observed turbine and all other turbines in the farm instead of absolute values between model and measurements. In this way, the uncertainty of the measurement chain could be reduced. The method uses pre-calculated power matrices which we call from now on "lookup tables" (LUTs). Different wake models or even combinations of wake model results can be used to provide results for these LUTs. But the method relies on measurements from a met mast which is often not available. Furthermore, with the increasing size of wind farms, the assumptions of one measurement position being representative of the whole offshore wind farm is not valid (Dörenkämper, 2015). Further investigations are necessary to obtain a reliable and automated method to detect underperformance at individual turbines in a wind farm.

The purpose of this paper is to present the results of extending the wind farm performance monitoring method of Mittelmeier et al. (2013) by using SCADA instead of met mast data. A new method to obtain representative environmental conditions and further optimization potential for wake models fine-tuned by SCADA data is presented, and an estimation of the uncertainty of these methods is given.
In Sect. 2 the general approach of the method by Mittelmeier et al. (2013) is recalled. A new approach to generate a virtual met mast from SCADA data is explained in detail in Sect. 2.1. The wake model optimizations are described in Sect. 2.2. A closer look at the uncertainties of the method especially in relation to the establishment of a virtual met mast is undertaken in Sect. 2.3. In Sects. 3, 4 and 5, results for a demonstration case are presented, followed by a detailed discussion and the final conclusions.

\section{Methods}

To detect underperformance of a wind turbine, we estimate the expected turbine power ratio $\pi$ (predicted power ratio) between the observed turbine and a reference turbine with a wake model for the actual condition and compare its result with the actual measured power ratio $\mu$. A deviation between $\pi$ and $\mu$ higher than a certain threshold indicates underperformance.

The performance monitoring model (Fig. 1) is based on two-dimensional LUTs. The user can choose any wake model or even a combination of different model results to provide power output $P_{\pi_{i, j}}$ values for different wind speed bins $i$ and wind direction bins $j$. The predicted power output $P_{\pi}$ is derived from the LUTs with linear interpolation, knowing the measured wind speed and wind direction.

It would be possible to use information about the turbulence intensity, pressure, temperature and humidity from additional devices to increase the dimensions of the power matrix, and this may add accuracy. As we are focusing on a monitoring method that uses only SCADA data, we will discuss and demonstrate one way to extract a useful wind speed and wind direction for this monitoring method in Sect. 2.1.

Commonly used power measurements are averages over 10 min periods. Due to the fact that there is a high scatter on power measurements for the same wind speed and wind direction bin, averaging a number $N$ of $10 \mathrm{~min}$ samples is necessary until the power value converges to a satisfactory degree. The power matrix and $N$ are derived in a pre-process as shown in Fig. 1, which gives an overview of the whole performance monitoring process.

The power of the wind turbine under observation $P_{\mathrm{ob}}$ is divided by the power of a reference wind turbine $P_{\text {ref. }}$. This leads to a normalized power curve with a much lower slope in a wide range of partial load (See Fig. 2) and therefore decreases sensitivity on wind speed measurement uncertainty. We define

$$
\begin{aligned}
& \mu=\frac{1}{N} \sum_{n=1}^{N} \frac{P_{\mu \mathrm{ob}_{n}}}{P_{\mu \mathrm{ref}_{n}}} \text { and } \\
& \pi=\frac{1}{N} \sum_{n=1}^{N} \frac{P_{\pi \mathrm{ob}_{n}}}{P_{\pi \mathrm{ref}_{n}}},
\end{aligned}
$$




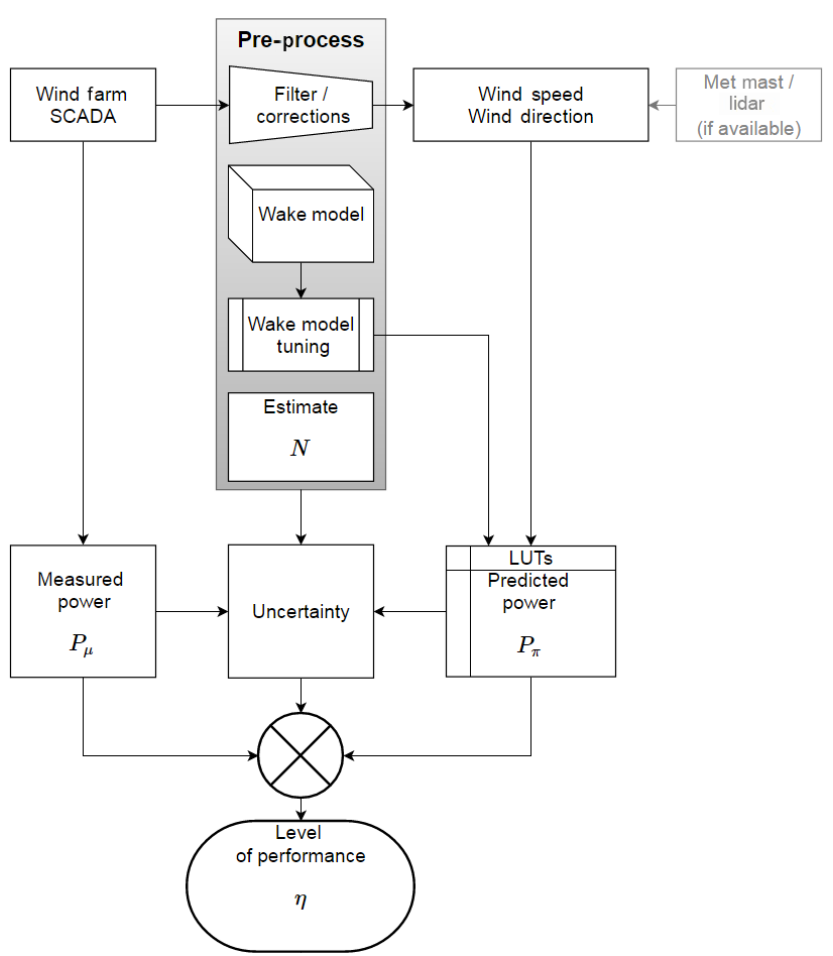

Figure 1. Flowchart of the performance monitoring model. Wind speed and wind direction are derived from SCADA data after an offset correction of each wind direction signal and outlier filtering. Wake model calculations and tuning as well as the estimation of the number $N$ of $10 \mathrm{~min}$ samples for averaging are preprocessed. $N, P_{\pi}$ and $P_{\mu}$ are input values for the uncertainty calculation. An underperformance indicator $\eta$ lower than the uncertainties indicates underperformance.

where $P_{\mu \mathrm{ob}}$ and $P_{\pi \mathrm{ob}}$ are the measured and predicted power of the observed turbine. $P_{\mu \mathrm{ref}}$ and $P_{\pi \mathrm{ref}}$ are the measured and predicted power of the reference turbine.

The underperformance indicator is defined as

$\eta_{\mathrm{ob}, \mathrm{ref}}=100 \%\left(1-\frac{\pi}{\mu}\right)$.

In this way, the underperformance interval range of the indicator is between $[0,-\infty]$. Non-operating turbine values have to be filtered out.

If $\eta_{\mathrm{ob}, \text { ref }}$ is larger than the uncertainty (Sect. 2.3), underperformance has been detected. This correlation is repeated for each combination of turbines, which leads to $n(n-1)$ results ( $n=$ number of turbines in the farm). This adds further confidence to the detection because an underperforming turbine will meet the uncertainty criterion several times.

\subsection{Determination of environmental conditions}

\subsubsection{Wind direction}

The first step is to derive a wind direction $\vartheta$ for each $10 \mathrm{~min}$ interval. For our monitoring model we are using the absolute wind direction signal from each turbine, which is defined as

$\vartheta=$ nacelle position + wind vane position.

The nacelle position is the angle between the rotor axis and a marking for true north. This marking is calibrated as part of the commissioning. But often this signal is not maintained well during operation because it has no effect on turbine performance. We need to apply an offset correction to this signal before using it. The wind vane position indicates the angle of the flow to the rotor axis. It directly provides a value for the yaw error. The turbine controller uses this signal to control the yaw activity.

Within the pre-process (Fig. 1) of the monitoring model, we estimate north, marking the offset for one turbine by checking the location of the maximum wake deficit with respect to true north. Then we compare the average wind direction between corrected turbines and neighboring turbines to estimate the remaining offset for all turbines. After applying this offset correction, the wind direction from all wind vanes is averaged in the complex plane to account for the wind direction discontinuity at the beginning/end of the value range, after removing outliers outside $\pm 1.5 \mathrm{IQR}$ (interquartile range).

\subsubsection{Wind speed}

Having determined an averaged wind direction, we are now able to derive the averaged free-flow wind speed. For this task we use the nacelle anemometry but only from wind turbines that are not affected by upwind turbines. To determine whether a turbine is affected by an upwind turbine or not, we use the specification for power curve measurements from the international standard (IEC 61400-12-1, 2005). Each turbine location is checked against all other turbine locations according to the averaged wind direction. This is done within a Cartesian coordinate system where $x$ represents the easting and $y$ is the northing (See Fig. 3). The wind turbine of interest $\mathrm{WT}_{\mathrm{i}}$ is located at the position $(x y)$, and the turbine wake is from the turbine $\mathrm{WT}_{0}$ at location $\left(x_{0} y_{0}\right)$. The width of the disturbed sector in degrees seen by the downwind turbine is defined according to IEC 61400-12-1 (2005) as

$\alpha=1.3 \arctan \left(2.5 \frac{D}{L}+0.15\right)+10^{\circ}$.

$D$ is the rotor diameter of the upwind turbine and $L$ the distance between the two turbines defined by Eq. (6).

$d_{x}=\left|x-x_{0}\right|$,

$d_{y}=\left|y-y_{0}\right|$,

$L=\sqrt{d_{x}^{2}+d_{y}^{2}}$.

With $\beta$ being the angle between the wake-inducing turbine and the northing and the wind direction $\vartheta$, the turbine wake 

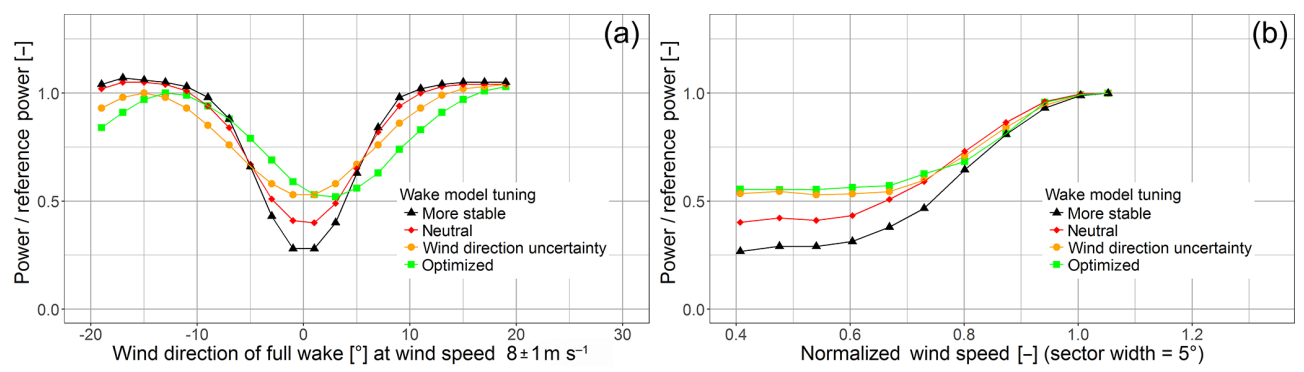

Figure 2. Impact of different key tuning aspects on the wake model results step by step. An increasing atmospheric stability increases the wake deficit (from red markers to black triangles). Wind direction uncertainty flattens the wake deficit (orange points), and a wind direction bias shifts the deficit horizontally (green squares). Panel (a) shows the power of the turbine in the wake divided by the power of a turbine in free-flow conditions as a function of the wind direction. Panel (b) displays the same power ratio as a function of the normalized wind speed (normalized power curve).

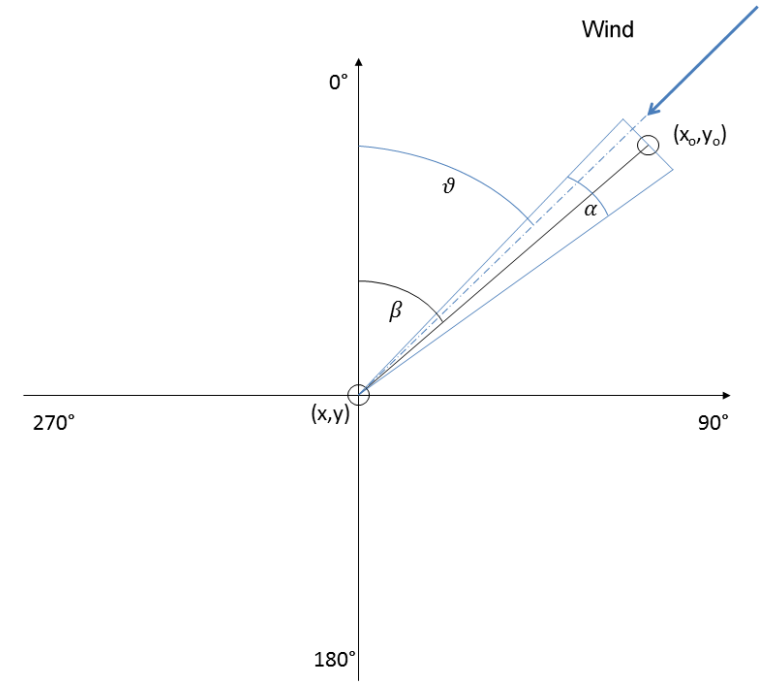

Figure 3. Determination of free-flow turbines for wind speed averaging. The turbine at $\left(x_{0}, y_{0}\right)$ produces a wake on the turbine at $(x, y)$ for the displayed wind direction $\vartheta . \beta$ is the angle between the orientation of the turbines and true north. $\alpha$ is the angle of the disturbed sector in accordance with IEC 61400-12-1.

indicator $\gamma$ can be described as

$\gamma= \begin{cases}|\beta+360-\vartheta|-\frac{\alpha}{2} & 0<\beta<90 \text { and } 270<\vartheta<360 \\ |\beta-360-\vartheta|-\frac{\alpha}{2} & 270<\beta<360 \text { and } 0 \leq \vartheta<90 . \\ |\beta-\vartheta|-\frac{\alpha}{2} & \text { else }\end{cases}$

The wind turbine of interest $\mathrm{WT}_{\mathrm{i}}$ is categorized as a waked turbine for $\gamma<0$. The wind speed for the virtual met mast is therefore the average of the subset of the nacelle anemometer signals from all wind turbines with $\gamma>0$.

\subsection{The wake model}

The wake model is a key factor in our performance monitoring method. Several benchmark tests have been published with a large variety of different models (Gaumond et al., 2012; Réthoré et al., 2013; Steinfeld et al., 2015). Research is still ongoing to further improve prediction accuracy of such models.

In Fig. 1 we highlight that the wake model and its tuning is part of the pre-process. The performance monitoring method itself is based on linear interpolation from the LUTs only. In Mittelmeier et al. (2015), three key parameters for the tuning of the wake model are identified (stability, wind direction uncertainty and wake drift). Figure 2 gives an example of how the different key parameters change the wake model results. The left plot shows the active power of a turbine in a wake normalized with a free-flow condition at $6.3 \mathrm{D}$ distance. The $0^{\circ}$ on the $x$ axis locates the full-wake situation according to the simulation. The right plot is a representation of the same data as a normalized power curve with wind speed on the $x$ axis normalized with the wind speed when wake effects fade away due to pitching activities of the upwind turbine.

In the first step, the wake model needs to be set up with the right atmospheric stability parameters. An increasing stability will cause higher wake losses and therefore shift the wake plot vertically down (from red markers to black triangles).

The next two steps are applied to the wake model results, which need to be calculated for a directional resolution of $0.5^{\circ}$ and for each wind speed bin of $1 \mathrm{~m} \mathrm{~s}^{-1}$. This resolution was proposed by Gaumond et al. (2014) for their method to account for measurement uncertainties related to the wind direction, which is the second key parameter in our tuning process. In Gaumond et al. (2014), three main sources of uncertainty are mentioned: the yaw misalignment of the reference turbine, the spatial variability of the wind direction within the wind farm and the variability of wind direction within the averaging of a $10 \mathrm{~min}$ interval. This causes a higher scatter in the data and leads to averaging effects that are not modeled in the simulation. In a post-process, each wind direction is averaged with weighted neighboring results. A Gaussian distribution with a standard deviation $\sigma_{a}$ has been proposed as a weighting function. The effect of this step is visualized in Fig. 2 (red markers are without and orange points 
Table 1. Type B uncertainties of the predicted power $P_{\pi}$.

\begin{tabular}{llll}
\hline $\mathrm{k}$ & Uncertainty component & Sensitivity $c_{k, i, j}$ & Uncertainty $u_{k, i, j}$ \\
\hline 1 & Wind speed estimation & $\left|\frac{P_{\pi i, j}-P_{\pi i-1, j}}{V_{i j}-V_{i-1, j}}\right|$ & 1 standard deviation of the averaged anemometers \\
2 & Wind direction estimation & $\left|\frac{P_{\pi i, j}-P_{\pi i-1, j}}{\vartheta_{i j}-\vartheta_{i-1, j}}\right|$ & 1 standard deviation of the averaged wind direction \\
\hline
\end{tabular}

are with $\sigma_{a}$ weighted averaging). In Mittelmeier et al. (2015), we could show that for the prevailing conditions at Ormonde wind farm $\sigma_{a}$ is a function of wind speed, decreasing with higher wind speeds.

Looking at the full wind rose for an annual energy production (AEP) estimation, the Gaussian averaging has little impact on the result (Gaumond et al., 2014). But the smaller the wind direction bin size, the larger the prediction error made by the wake model. Hence, it is crucial for our monitoring method to increase accuracy for smaller wind direction bin sizes which will decrease the uncertainty of the method.

The third tuning parameter applies a simple offset on the wind direction of the LUTs to account for a drift of the wake. We call this phenomenon from here on "wake drift". Fleming et al. (2014) studied the effects of active wake control, and in his baseline simulation (no yaw error) a small wake drift to the right can be observed when looking downwind. In the large eddy simulation (LES) study of Vollmer et al. (2016), the wake drift increases from neutral to stable conditions also for $0^{\circ}$ yaw angle. Gebraad $(2014$, p. 86) gives an explanation for the observations from the simulations by Fleming et al. (2014). The flow reacting on the rotation of the rotor causes the wake to rotate counterclockwise (looking downstream). Higher wind speeds from the upper layer are transported downwards (on the left side) and lower wind speeds from the lower layer are pushed upward on the right side of the wake. As a result the velocity deficit at the right part of the wake increases, so the wake deflects to the right.

Marathe et al. (2016) could show in their field measurement campaign with a dual-Doppler radar the wake drifting to the right. But in the far wake they registered a movement to the left. The authors state the hypothesis that this contradicting phenomenon may be caused by atmospheric streaks. In an offshore field experiment by Beck et al. (2015), further evidence is provided that wakes are moving out of the center line.

\subsection{Uncertainties and underperformance criteria}

It is essential to understand the uncertainties of the method to judge the confidence in underperformance detection. Any false alarm can cause unnecessary trouble shooting.

For this evaluation, we follow the "Guide to the expression of Uncertainties in Measurements" (JCGM, 2008), which distinguishes between statistical Type A and instrumental Type B uncertainties. The important measurands of

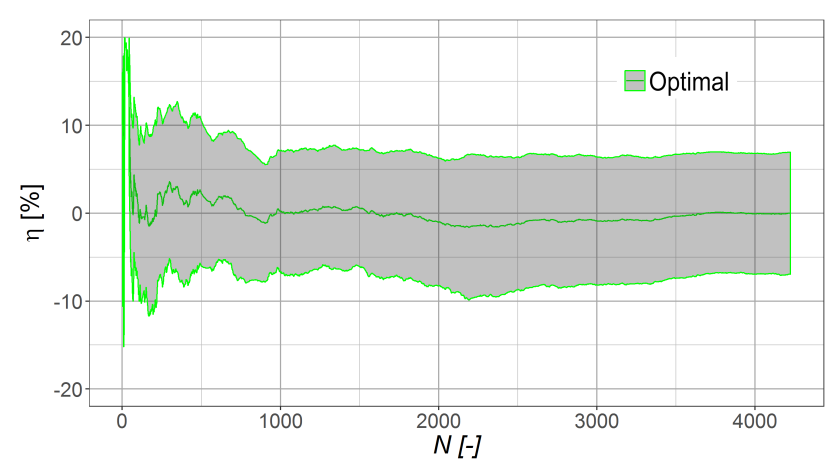

Figure 4. Underperformance indicator $\eta$ with uncertainty margin as a function of the number of measurement values $N$. Derived with the calibrated model at a turbine in triple wake.

the method are the measured power and the predicted power for each wind turbine under observation and for reference $\left(P_{\mu \mathrm{ob}}, P_{\mu \mathrm{ref}}, P_{\pi \mathrm{ob}}, P_{\pi \mathrm{ref}}\right)$. For the measured power $P_{\mu}$, we only use Type $\mathrm{B}$ because each measurand is obtained from different environmental conditions and therefore statistical Type A uncertainties are not applicable. The combined uncertainty can be derived with Eq. (8):

$u_{c}(P)=\sum_{k=1}^{K}\left(c(P)_{k} u_{k}\right)^{2}$,

where $c_{k}$ is the sensitivity factor and $u_{k}$ the uncertainty of the $k$ th component of the measurement chain of length $K$. For the predicted power $P_{\pi}$, we are using a combined uncertainty, with statistical Type A uncertainties being the experimental standard deviation of the mean from the difference between wake model predictions and measurements and Type B being uncertainties which allows inferences to be made from the instrument devices to estimate wind speed and wind direction. Table 1 shows the uncertainty components of the predicted power $P_{\pi}$ and provides the sensitivity factors. $P_{\pi i, j}$ is the power value in the matrix referring to the wind speed bin $i$ and the wind direction bin $j . V_{i, j}$ is the wind speed and $\vartheta_{i, j}$ the wind direction of the element. In Table 2 , the corresponding components for the uncertainty of the measured power $P_{\mu}$ are listed.

Results from the offshore wind accelerator (Clerc et al., 2016) provide a range of 2.5 to $5 \%$ combined uncertainty for power curve verification based on a measurement chain 
Table 2. Type B uncertainties of the measured power $P_{\mu}$ (values as suggested by IEC 61400-12-1).

\begin{tabular}{llll}
\hline$k$ & Uncertainty component & Sensitivity $c_{k, i, j}$ & Uncertainty $u_{k, i, j}$ \\
\hline 1 & Current transformer & 1 & $0.0043 P(\mathrm{~kW})$ \\
2 & Voltage transformer & 1 & $0.003 P(\mathrm{~kW})$ \\
3 & Power transducer & 1 & $0.003 P_{\text {rated }}(\mathrm{kW})$ \\
4 & Power data acquisition & 1 & $0.001 P_{\text {rated }}(\mathrm{kW})$ \\
\hline
\end{tabular}

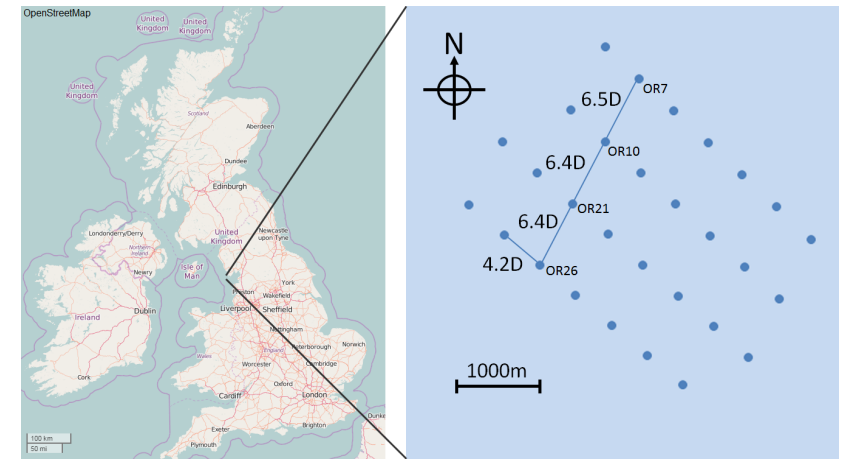

Figure 5. Layout of wind farm Ormonde. The 30 turbines of $5 \mathrm{MW}$ class are located in the Irish Sea $10 \mathrm{~km}$ west of the Isle of Walney. For a wind direction of $207^{\circ}$ the single wake, double wake and triple wake behind OR26 have been selected as underperformance demonstration cases.

that includes a met mast and all its devices. The use of lidar extends the range up to approximately $7 \%$. In our case, the wake model will add further uncertainties which would lead to even higher values and therefore yields an unacceptable rate for underperformance detection. To lower this impact, the monitoring method is based on normalized measurements and normalized predictions. An error at the estimated wind speed has a much lower impact on the ratio of the power of two turbines than on their absolute power performance. The uncertainty for Eq. (1) can be described as

$$
\begin{aligned}
u(\mu) & =u\left(\frac{P_{\mu \mathrm{ob}}}{P_{\mu \mathrm{ref}}}\right) \\
& =\frac{P_{\mu \mathrm{ref}}}{P_{\mu \mathrm{ob}}} \sqrt{\left(\frac{u_{c}\left(P_{\mu \mathrm{ob}}\right)}{P_{\mu \mathrm{ob}}}\right)^{2}+\left(\frac{u_{c}\left(P_{\mu \mathrm{ref}}\right)}{P_{\mu \mathrm{ref}}}\right)^{2}} .
\end{aligned}
$$

The equation is equivalent for $u(\pi)$ and is applied to each 10 min sample. With the two uncertainties $u(\mu)$ and $u(\pi)$ being independent, the standard propagation of errors for $\eta$ can be simplified according to $\mathrm{Ku}$ (1966) to the following equation:

$u^{2}(\eta)=\left(\frac{\partial \eta}{\partial \mu}\right)^{2} u^{2}(\mu)+\left(\frac{\partial \eta}{\partial \pi}\right)^{2} u^{2}(\pi)$

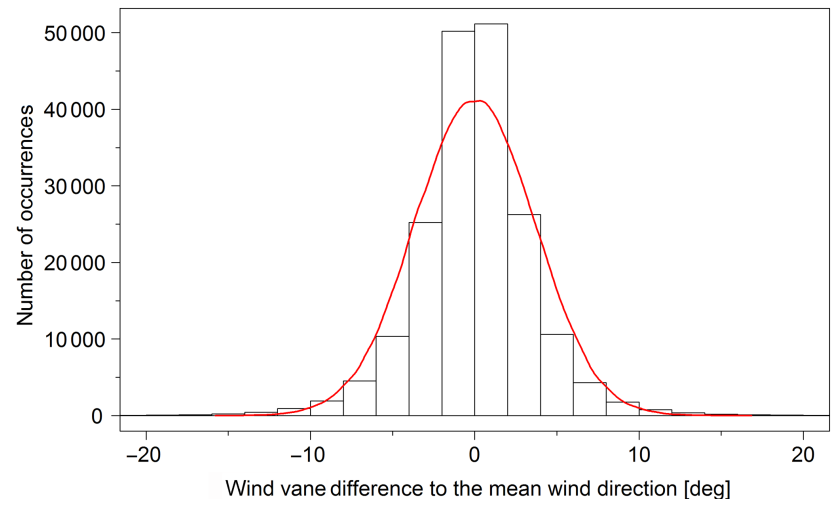

Figure 6. Estimation of the uncertainty of the artificial wind direction. Histogram of the deviation of 30 individual wind vanes from the average wind direction for the full data set filtered for wind speeds $>5 \mathrm{~m} \mathrm{~s}^{-1}$ with a sector of $30^{\circ}$ centering on the full-wake condition. The red curve represents a Gaussian fit with a standard deviation of $3.6^{\circ}$.

which leads to an uncertainty in $\eta$ of

$u(\eta)=\frac{100}{\mu} \sqrt{u^{2}(\pi)+\left(\frac{\pi}{\mu}\right)^{2} u^{2}(\mu)}$.

The uncertainty derived by Eq. (11) can be displayed as a bandwidth around the underperformance indicator $\eta$, visualized in Fig. 4. Its magnitude is dependent on the sample size $N$. In Fig. 4 we obtain an approximately $7 \%$ uncertainty on the performance ratio for $N>1000$. The confidence level is 1 standard deviation, which is considered to be acceptable for underperformance detection.

In the next step we need to estimate the required number of power samples $N$ for averaging (see Eqs. 1 and 2). This is directly linked with the earliest point in time when underperformance can be detected. We define this point as having a lower prediction error (with the optimal turbine operation model) than the prediction error derived by the model with the erroneous data taking the uncertainty into account.

\section{Results and demonstration}

We have chosen the Ormonde wind farm to demonstrate the new method. The 30 turbines have a rated power of $5 \mathrm{MW}$ and are owned by Vattenfall. The wind farm is located in the Irish Sea, $10 \mathrm{~km}$ west of the Isle of Walney. 


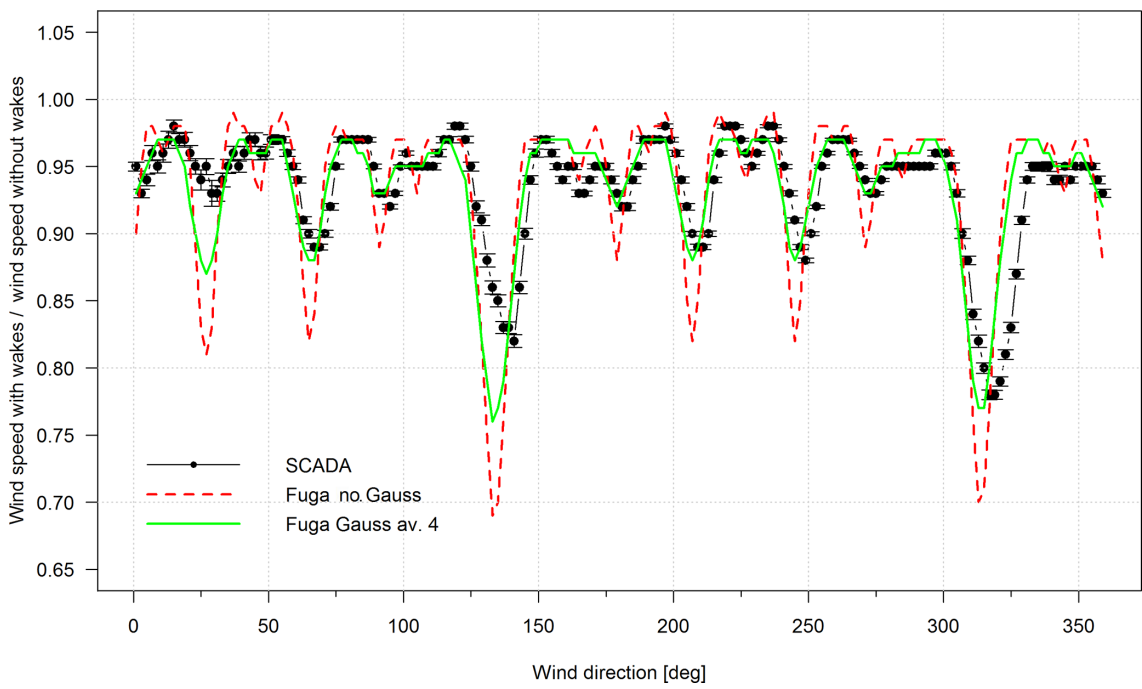

Figure 7. Wind-farm-averaged wind speed with wake effects normalized, with wind-farm-averaged wind speed without wake effects plotted versus averaged wind farm wind direction. Black dots show the measurements from SCADA, and the green solid line represents the results from Fuga with a Gauss averaging for a standard deviation of $4^{\circ}$. An offset of the wind direction between the model and SCADA can be observed. At $207^{\circ}$ the offset is approximately $2.2^{\circ}$, and it increases up to $5^{\circ}$ for wind directions $\left(132\right.$ and $\left.312^{\circ}\right)$ with the largest wake effects. An explanation and correction for this wake drift is proposed in Sect. 2.2.

The farm layout displayed in Fig. 5 is structured in a regular array, which allows the comparison of several wake situations. The closest turbine spacing is in the range of 4.1 to $4.3 \mathrm{D}$ along the four rows orientated from northwest to southeast. We select a more frequent wind direction from south-southwest where multiple columns of four turbines are aligned with a distance ranging from 6.3 to $6.5 \mathrm{D}$. To simplify the demonstration of underperformance detection, we focused on single-wake, double-wake and triple-wake conditions behind turbine OR26 for a south-southwesterly wind direction and a sector of $30^{\circ}$ around the full-wake situation. Two years of $10 \mathrm{~min}$ SCADA data were used to set up the performance monitoring model.

\subsection{Environmental condition of demonstration wind farm}

\subsubsection{Wind direction}

In our example, we have averaged up to 30 corrected wind direction signals for each 10 min interval. The variation among the individual signals provides an uncertainty estimate for this artificial wind direction. In Fig. 6, a histogram of the full data set of 2 years, with each count being the difference between a single-vane measurement and the corresponding mean wind direction for the averaged period, is visualized. This variation can be nicely described by a Gaussian distribution with a standard deviation of $3.6^{\circ}$. This value is used for the uncertainty of the wind direction Table 1 refers to.

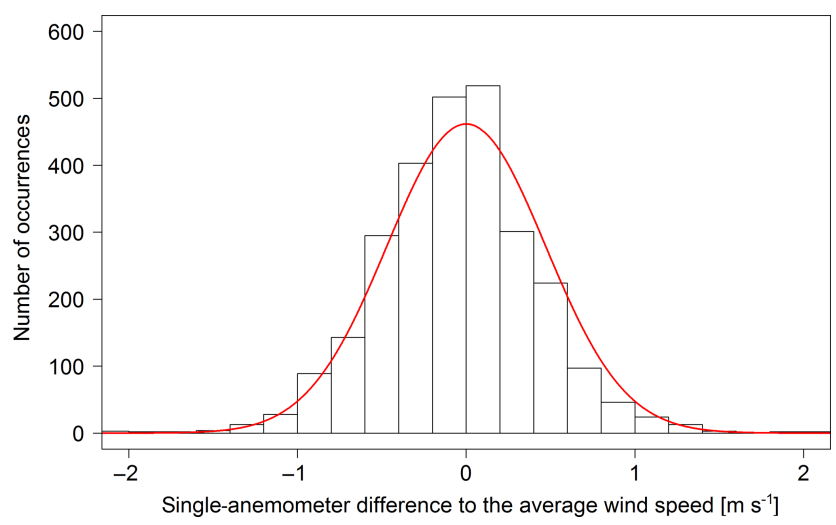

Figure 8. Estimation of uncertainty of the artificial wind speed. Histogram of the wind speed difference between a single anemometer and the average wind speed of all free-flow anemometers. The displayed Gaussian distribution (red line) has the standard deviation of $0.46 \mathrm{~m} \mathrm{~s}^{-1}$. A sector of $30^{\circ}$ centering full-wake alignment has been selected.

\subsubsection{Wind speed}

Figure 7 demonstrates the quality of the virtual met mast derived with the methodologies described in Sect. 2.1.1 and 2.1.2. The average wind speed of all nacelle anemometers is normalized by the averaged nacelle anemometer wind speed of the wake-free subset. The full data are binned into $2^{\circ}$ and plotted against the averaged wind direction. The errors bars indicate the experimental standard deviation of the mean (JCGM, 2008). We obtain quite a good agreement with the Fuga model, which was used with $\sigma_{a}=4^{\circ}$. So far, there is 

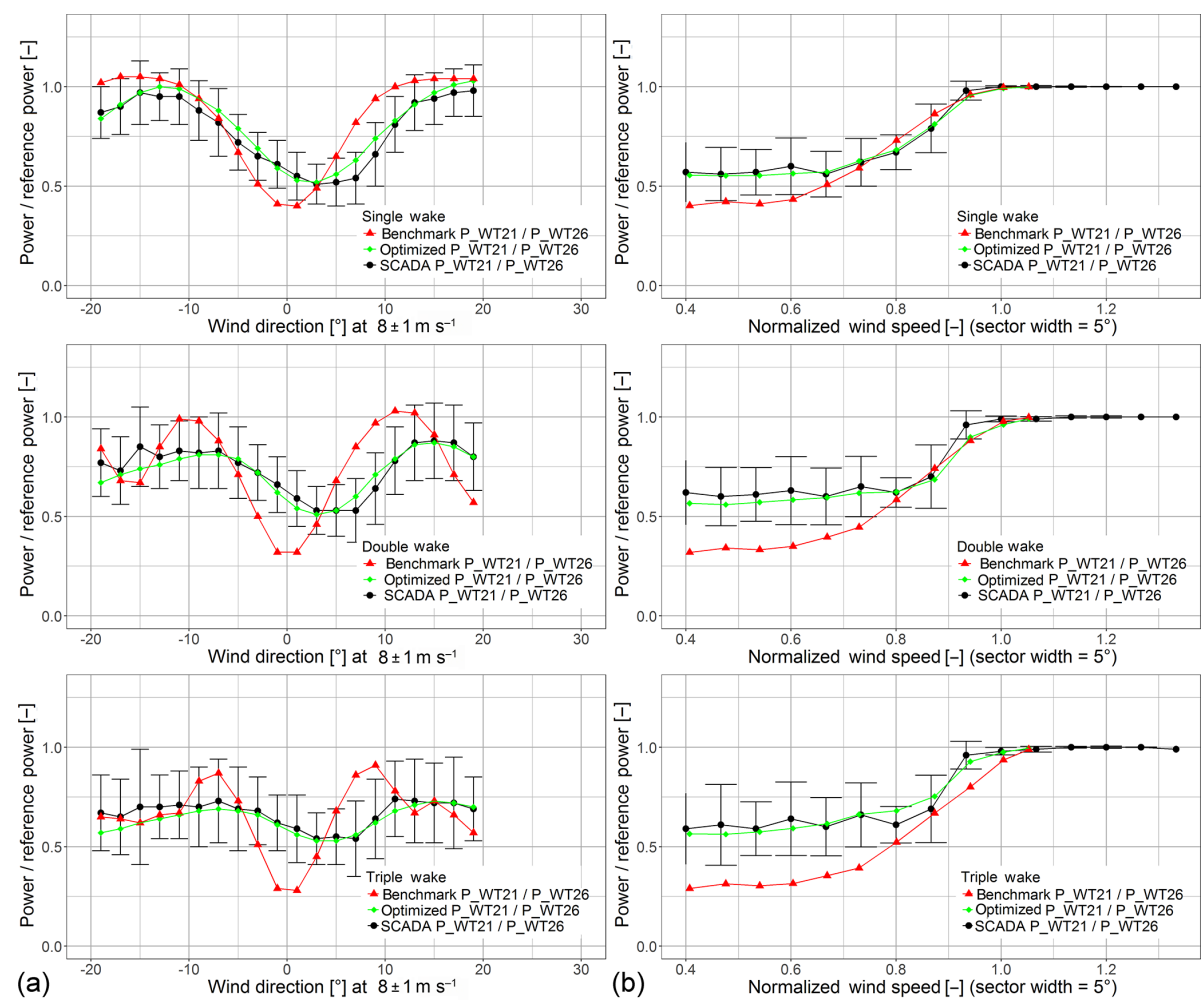

Figure 9. Tuning of the wake model results. (a) Power normalized by the power of the free-flow turbine as a function of the wind direction centered at full wake for $8 \pm 1 \mathrm{~m} \mathrm{~s}^{-1}$ wind speed. (b) Power normalized by the power of the free-flow turbine as a function of the wind speed normalized by wind speed at rated power for the waked turbine. Black dots represent the measured and binned SCADA data with error bars of 1 standard deviation. The red triangles show wake model results with Fuga standard settings $\left(\zeta_{0}=0\right.$, no Gaussian averaging), and the green diamonds provide the tuned results. $\left(\zeta_{0}=2.72 \times 10^{-7}\right.$, Gaussian averaging as a function of the wind speed and applying the wind direction offset to account for the wake drift).

no instruction available on how to determine this standard deviation, which should take wind direction uncertainty into account (Gaumond et al., 2014). We have chosen this value because of quite a nice fit with the SCADA data. The coefficient of determination of a linear regression between the wind speed of the standard model results (red dashed line) and the SCADA measurements equals $R^{2}=0.96$. The improved model (green solid line) gives an $R^{2}=0.97$.

When considering the demonstration sector of $30^{\circ}$ around the full-wake alignment behind wind turbine 26 , the freeflow wind speed can also be described by a Gaussian distribution (Fig. 8), with a standard deviation of $0.46 \mathrm{~m} \mathrm{~s}^{-1}$.

This information is important for the investigation of the uncertainties Table 1 refers to.

\subsection{Wake model}

For the demonstration of the described method, we used the Fuga wake model, which uses linearized Reynolds-averaged Navier-Stokes equations developed by Ott et al. (2011). With the second version of the software, new features were added (Ott and Nielsen, 2014) to account for different atmospheric stabilities and for wind direction uncertainties. The results for this paper have been produced with Fuga version 2.8.4.1. We have chosen this wake model for two reasons: firstly, there is already a confident number of validations with measurements published (Gaumond et al., 2012; Mortensen et al., 2013; Steinfeld et al., 2015) and secondly, the Gaussian averaging feature described by Gaumond et al. (2014) is already implemented.

To get a more reliable monitoring method, we need to calibrate the wake model settings and compare several different calculation results with measured SCADA data based on the established virtual met mast. The wake model is supposed to provide a two-dimensional LUT (wind direction, wind speed) for each turbine. Further dimensions such as stability may improve the accuracy, but research and validation for these models are still ongoing. Therefore, our calibrated model has to be representative of the average annual conditions. Two full years of SCADA data are used for this task.

We identify three steps to obtain a better match between the power modeled by the wake model Fuga and the measurements. Firstly, the standard deviation $\sigma_{a}$ to account for the wind direction uncertainty is found to decrease with increasing wind speed. Secondly, the dimensionless parameter 


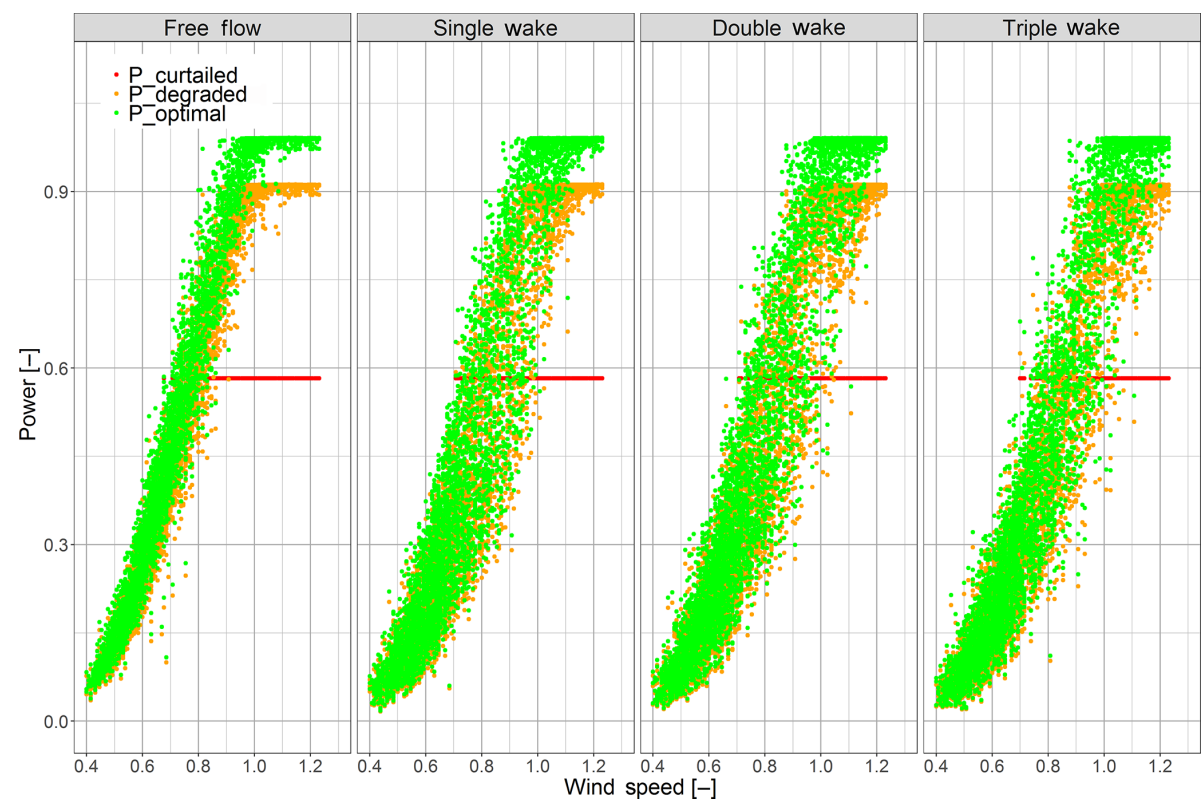

Figure 10. Scatterplot with normalized power as a function of the normalized wind speed for four turbines in one row with two error test cases. Green dots are the measured power values and represent optimal operation. The $8 \%$ degradation of the power output is shown with yellow dots. A curtailment at $58 \%$ is shown in red.

to model the effect of atmospheric stability $\zeta_{0}=2.72 e-7$ is set to stable conditions, and thirdly, the center of the wake drifts towards the right side when traveling downwind. Approximately $2.5^{\circ}$ in the single wake and an additional $1^{\circ}$ is added with every turbine, adding an additional wake to the flow. This results in a total offset of $4.5^{\circ}$ for the triple wake referenced to the artificial wind direction from the virtual met mast (Sect. 2.1.1). One possible explanation for this behavior is the fact that the upward-moving blade diverts the flow with higher wind speeds downwards to regions with lower wind speeds and the downward-moving blade causes the opposite. This results in a higher wind speed on the left side than on the right side of the wake and leads to a drift of the wake center. A second explanation can be derived from the Coriolis force, which leads to an increased force to the right (Northern Hemisphere) on accelerating air particles. We cannot fully rule out the possibility of an unwanted yaw misalignment as the uncertainties within this process of aligning the turbine lie within $3^{\circ}$ (IEC 61400-12-2, 2013). But a single-wake drift of $2.5^{\circ}$ is also within the simulation results for $0^{\circ}$ yaw misalignment under stable conditions (Vollmer et al., 2016), whereas neutral and unstable conditions show no drift or even a very small drift in the opposite direction.

The column of turbines behind turbine OR26 has been selected for the validation of the wake model settings. The benchmark is simulations for neutral conditions with none of the post-processings mentioned in Sect. 2.2 to take wind direction uncertainty, atmospheric stability and wake drifts into account. Figure 9 demonstrates the improvement of model prediction and its capabilities for single-wake, double- wake and triple-wake situations. The left column visualizes wake deficit plots where the power has been normalized with the free-flow turbine, as a function of the wind direction, centered on the full wake. The data are filtered for a wind speed of $8 \pm 1 \mathrm{~m} \mathrm{~s}^{-1}$. The right column is normalized wake power curves. The power, normalized with free-flow power, is shown as a function of the wind speed, normalized with wind speed at rated power for the turbine in the wake. These data are filtered for a wind direction sector of $5^{\circ}$. The optimized simulation results (green diamonds) follow the SCADA data (black dots) much more closely than the benchmark case (red triangles). The error bars indicate 1 standard deviation of the measured SCADA data at each bin. The three fine-tuning steps decreased the power prediction error in a full wake with $\pm 5^{\circ}$ sector width from 7 to $1.5 \%$ (Mittelmeier et al., 2015) for the case presented.

Having now obtained an optimized wake model, the first two steps of the pre-process (Fig. 1) are accomplished and the matrices for the "predicted power" can be established. In the next section, the detection of underperformance will be demonstrated with two test cases.

\subsection{Demonstration case}

Two years of SCADA data are contaminated with two different error types. The first manipulation simulates a degradation of $8 \%$ of its power production; to do this, the original data set that was used to calibrate the model is multiplied by 0.92. According to the findings in Sect. 2.3, a degradation of $8 \%$ is just high enough to distinguish it from the uncer- 


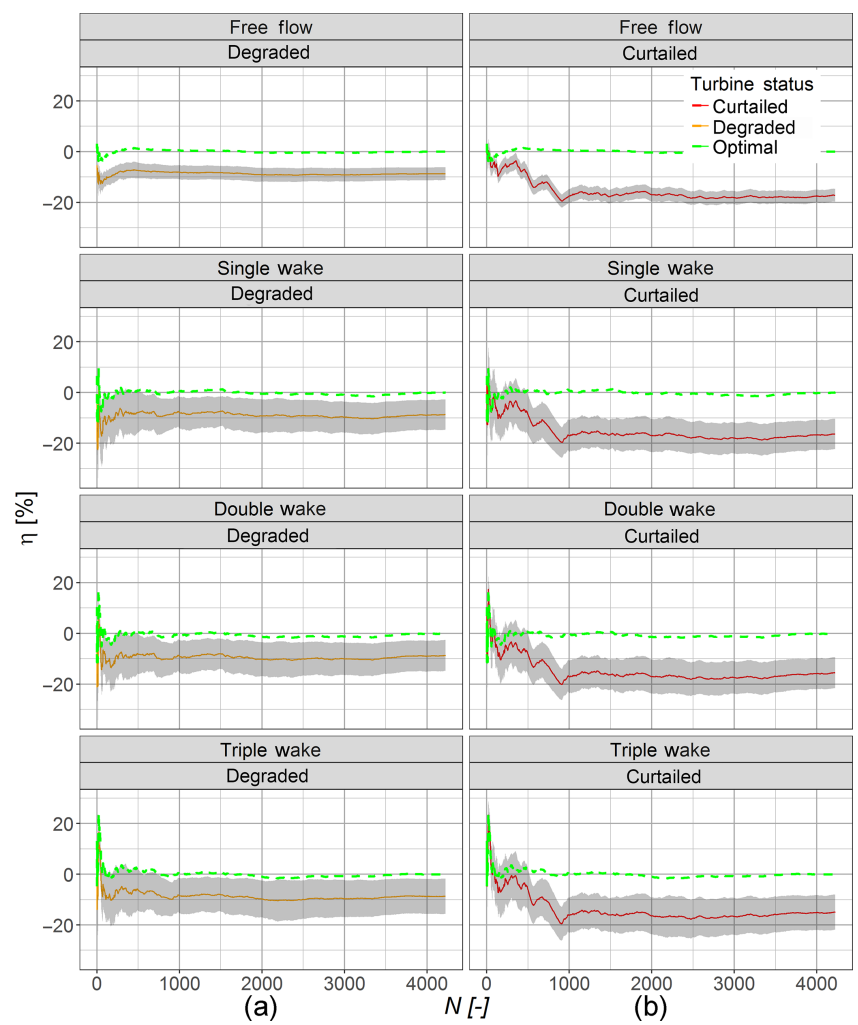

Figure 11. Underperformance detection for curtailment (b) and degradation (a) at turbines with different levels of wake influence. The displayed values represent the underperformance indicator $\eta$ as a function of the number of values $N$. We highlight the first time of underperformance detection when the green dotted line is outside of the grey uncertainty bandwidth.

tainties of a turbine in triple wake. The second test case is a simple power curve curtailment at $58 \%$ rated power.

In Fig. 10 the normalized power as a function of the normalized wind speed is shown in a scatterplot. The colored points in green represent correct turbine performance $\left(P_{\text {optimal }}\right)$. The yellow dots $\left(P_{\text {degraded }}\right)$ describe the degradation, and the red dots $\left(P_{\text {curtailed }}\right)$ are the data with the curtailment. Measurements from above rated wind speed are removed to concentrate on the part of the power curve where underperformance is more difficult to detect.

Below $5 \mathrm{~m} \mathrm{~s}^{-1}$ we find a strong increase in wind direction variation among the turbines compared to the artificial wind direction from the virtual met mast. This variation increases the uncertainty of the model, and therefore wind speed below $5 \mathrm{~m} \mathrm{~s}^{-1}$ is filtered.

To further increase the certainty of the result, we calculate the underperformance indicator for each turbine with all other possible combinations of reference turbine. For the whole wind farm of 30 turbines, this leads to 870 combinations. For simplification in this demonstration, we are only focussing on the four turbines in the row behind turbine OR26.

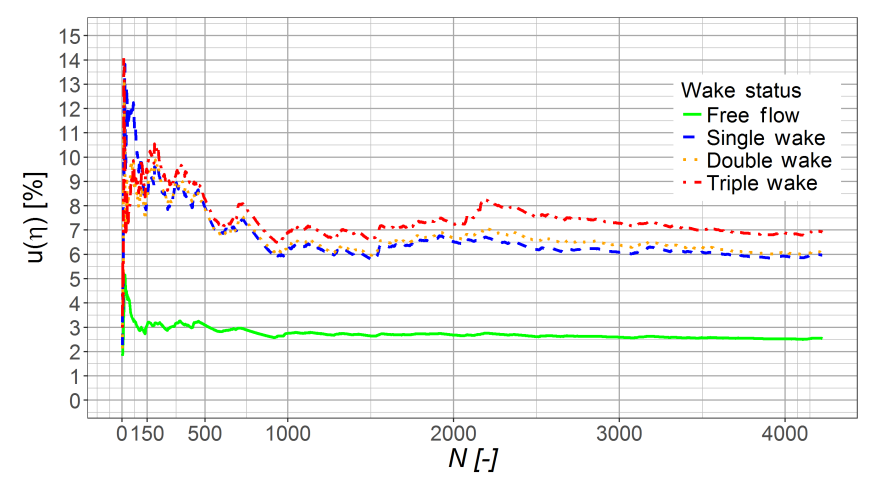

Figure 12. Uncertainties for the underperformance indicator $u(\eta)$ as a function of $N$ values for free-flow, single-wake, double-wake and triple-wake situations. Uncertainties for free-flow conditions (green) are much lower than the uncertainties for the waked turbines.

First, we need to estimate the required number of power samples $N$ for averaging. This can be visualized in Fig. 11 . The graphs present the accumulated level of underperformance $\eta$ as a function of the number of samples ( $N$ values). The data with the error appear as a solid line with a grey uncertainty margin. The dashed green line represents the data with the turbine in optimal operation. The lowest quantity $N$, where the "optimal" (green dashed) line confirms a lower $\eta$ than the border of the grey area, is the point where we highlight underperformance with sufficient certainty. Figure 11 demonstrates the two test cases with a turbine under curtailment at different wake situations and the corresponding situation for a degraded turbine. A wake model bias is corrected in such a way that the results for the optimal turbine prediction with the full 2 years of data equals zero.

In Table 3 we have listed the $N$ values which it is necessary to measure in each case until underperformance can be detected with sufficient certainty. They can be translated into hours by $N / 6$ as we are using $10 \mathrm{~min}$ averages.

Figure 12 is a graphical representation of the development of uncertainty with an increasing number of averaging data. The free flow has a comparatively low uncertainty in comparison with the three wake situations. All four graphs have higher uncertainty in the beginning, which quickly decreases with increasing $N$. At approximately $N=150$ the uncertainty of all three wake states has dropped at least once below $8 \%$. With $150<N<500, u(\eta)$ is still very unstable and stretches between 8 and $10 \%$.

A clear additional drop, even below $7 \%$, can be seen from $500<N<1000$. Beyond $N>1000, u(\eta)$ stabilizes towards a more and more horizontal line. In Table 4 the corresponding uncertainty for the estimated first time of detection is listed.

The power curve scatterplot of all four wake conditions with the number of quantities necessary for detection are visualized in Fig. 13. 


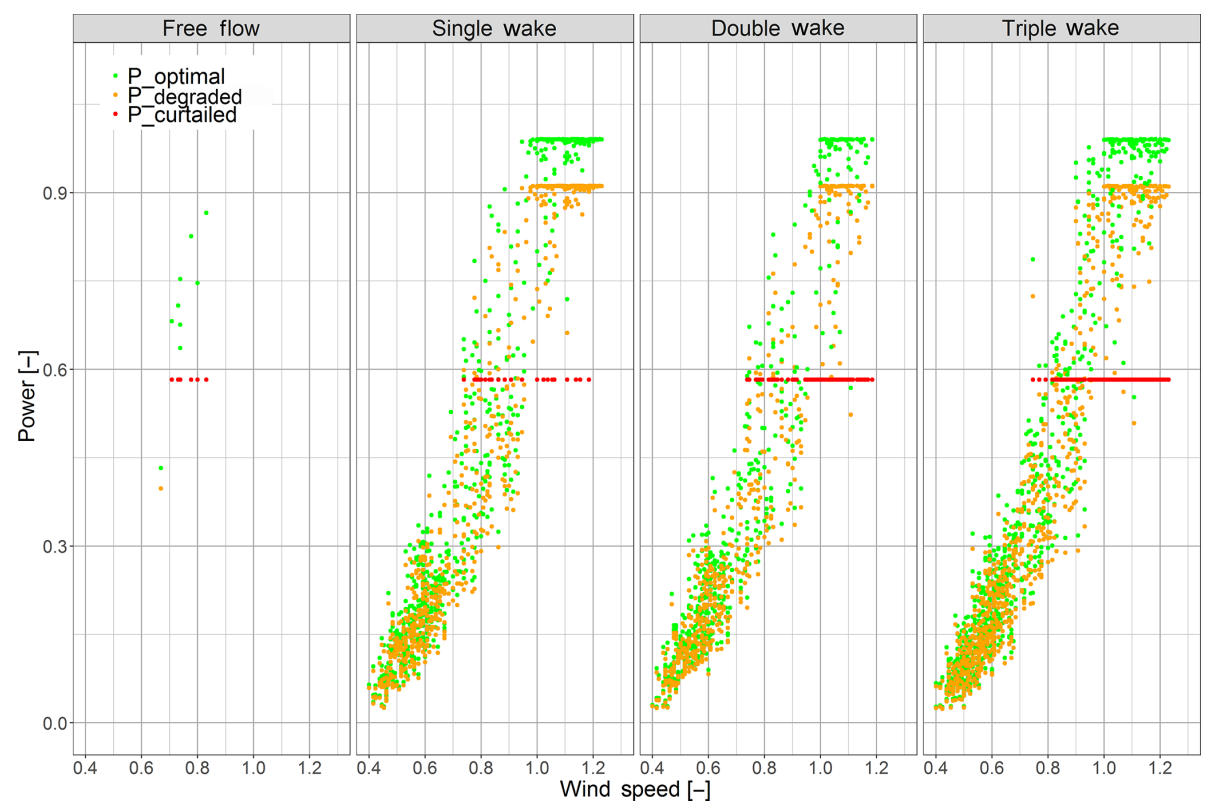

Figure 13. Scatterplot of each turbine's normalized power curve. The quantity $N=754$ equals the estimated sample size for the first detection of degradation at a turbine in a triple-wake situation.

Table 3. $N$ values to the first detection of underperformance with a certainty of 1 standard deviation. Values in brackets indicate $N$ with wind speeds above the curtailment.

\begin{tabular}{lrr}
\hline Wake situation & $N_{\text {degradation }}(-)$ & $N_{\text {curtailment }}(-)$ \\
\hline Free flow & 1 & $51(8)$ \\
Single wake & 577 & $149(28)$ \\
Double wake & 502 & $501(106)$ \\
Triple wake & 754 & $655(164)$ \\
\hline
\end{tabular}

Table 4. Uncertainty $U$ at quantity $N$ of the first detection of underperformance with a certainty of 1 standard deviation.

\begin{tabular}{lrr}
\hline Wake situation & $U_{\text {degradation }}(\%)$ & $U_{\text {curtailment }}(\%)$ \\
\hline Free flow & 1.8 & 3.6 \\
Single wake & 6.9 & 7.9 \\
Double wake & 7.8 & 7.8 \\
Triple wake & 7.8 & 7.3 \\
\hline
\end{tabular}

\section{Discussion}

The model was able to detect the selected demonstration error cases after a certain averaging time. With the proposed sources of uncertainty and the described method to obtain a combined level, a very clear increase in uncertainty can be seen from free-flow to wake condition cases. The reason for this behavior lies in the normalization procedure. The largest source of uncertainty is usually the wind speed measurement, followed by the wind direction measurement (Type B uncer- tainties). Looking at the sensitivity factor for both readings, which is based on the slope of the quotient between neighboring normalized LUTs cells, it approximately equals zero for the free-flow case. Therefore, only Type A uncertainties are left, which quickly decrease with an increasing number of measured values $N$.

In our example, the curtailment took less than 170 values to be detected (see Table 3). This, of course, is highly dependent on the wind distribution. The wind has to be high enough to force the turbine into underperformance. At rated wind, detection is much faster than at wind varying around the power limitation. The right column in Table 3 shows the total values $N$ and in brackets the values considering only wind speeds high enough to force the turbine into curtailment. The $N$ values in Table 3 increase with each additional wake added to the flow. Furthermore, the figures show that curtailments (values in brackets) can be detected earlier than degradation.

The tuning of the wake model is an essential part of the method. The key tuning parameters have been estimated by trying to obtain the best fit with the SCADA data. This is a clear weak point of the method, and further investigations are necessary to find ways to predict the right settings without measured data. Without such tuning, each of these parameters will contribute as an additional source of uncertainty and therefore reduce the accuracy. A further improvement could be to extend the dimensions of the LUTs with atmospheric stability. Dörenkämper et al. (2012) was able to show that the influence on the development of wind turbine wakes is measurable. A link between SCADA data and atmospheric 
stability would be needed. An investigation is planned for future work.

The sensitivity of the underperformance indicator $\eta$ states the measured power correlation in the denominator of Eq. (3). In this way the interval range increases from [0, $-100]$ to $[0,-\infty]$. Division with 0 is prevented by filtering non-operating conditions. The increased interval leads to a higher sensitivity and therefore further reduces the $N$ values for the first underperformance detection.

Using wind speed and wind direction measurements derived from a large number of devices can lead to acceptable levels of uncertainties although each single device for itself has comparably high uncertainties as described in more detail in the power verification standard using nacelle anemometry (IEC 61400-12-2, 2013). The stated uncertainties for wind speed and wind direction may be sufficient for the relative comparison to detect underperformance between turbines, but they do not meet the requirements for an absolute performance validation according to IEC 61400-12-1 (2005) or IEC 61400-12-2 (2013). One could perform power curve verification tests in accordance with the mentioned standards at turbines where they are applicable, and those turbines that are reference turbines in the monitoring method would increase the confidence in underperformance detection. At least for the concurrent period.

\section{Conclusion}

A method for offshore wind farm power performance monitoring with SCADA data and advanced wake models was introduced. Wind speed and wind direction were extracted from all devices in the wind farm to obtain a global measurement for the whole wind farm. In this way, the level of uncertainty could be lowered compared to a single-nacelle measurement. Furthermore, the uncertainties in performance level prediction could be reduced by normalization and cross-reference correlations. A suitable wake model was chosen, calibrated with SCADA data and used in a demonstration case. A procedure to determine the optimal number $N$ of 10 min samples to detect underperformance with sufficient certainty has been presented. Here, the method was capable of detecting a degradation of $8 \%$ in a triple-wake situation with the confidence of 1 standard deviation. The described method can be used after a wake model calibration with approximately 2 years of wind farm SCADA data. This can enable real-time monitoring from then on for the rest of the operational lifetime.

Data availability. None of the data are publicly available.

Competing interests. The authors declare that they have no conflict of interest.
Acknowledgements. The work presented is partly funded by the Commission of the European Communities, Research DirectorateGeneral, as part of the project "ClusterDesign" (project no. 283145 (FP7 Energy)).

We would like to thank Vattenfall Wind Power and Senvion SE for making this investigation possible.

Furthermore, we would like to thank the $\mathrm{R}$ Core Team for developing the open-source language R (R_Core_Team, 2015).

Edited by: Gerard J. W. van Bussel

Reviewed by: three anonymous referees

\section{References}

Albers, A.: Efficient wind farm performance analysis, in: 7th German Wind Energy Conference, DEWEK, 20-21 October, Deutsche WindGuard Consulting GmbH, Wilhelmshaven, Germany, 2004a.

Albers, A.: Relative and Integral Wind Turbine Power Performance Evaluation, in: Proceedings of the European Wind Energy Conference and Exhibition, 22-25 November, London, UK, 2004b.

Albers, A., Klug, H., and Westermann, D.: Power performance verification, in: Proceedings of the European Wind Energy Conference, 657-660, 1-5 March, Nice, France, 1999.

Beck, H., Trujillo, J. J., Wolken-möhlmann, G., Gottschall, J., Schmidt, J., Peña, A., Gomes, V., Lange, B., Hasager, C., and Kühn, M.: Comparison of simulations of the far wake of alpha ventus against ship-based LiDAR measurements, in: RAVE Conference, 13-15 October, Bremerhaven, Germany, 2015.

Carvalho, H. and Guedes, R.: Wind Farm Power Performance Test, in: Scope of the IEC 61400-12-3, Wind Power Expo, Chicago, USA, 2009.

Clerc, A., Stuart, P., Cameron, L., Feeney, S., and Fnc, I. C.: Results from the Offshore Wind Accelerator (OWA) Power Curve Validation using LiDAR Project, in: Wind Europe Workshop "Analysis of operating wind farms", Bilbao, Spain, 2016.

Dörenkämper, M.: An investigation of the atmospheric influence on spatial and temporal power fluctuations in offshore wind farms, University of Oldenburg, Oldenburg, 2015.

Dörenkämper, M., Tambke, J., Steinfeld, G., Heinemann, D., and Kühn, M.: Influence of marine boundary layer characteristics on power curves of multi megawatt offshore wind turbines, in: Proceedings of 11th German Wind Energy Conference, DEWEK, 2012.

Fleming, P. A., Gebraad, P. M. O., Lee, S., van Wingerden, J. W., Johnson, K., Churchfield, M., Michalakes, J., Spalart, P., and Moriarty, P.: Evaluating techniques for redirecting turbine wakes using SOWFA, Renew. Energ., 70, 211-218, doi:10.1016/j.renene.2014.02.015, 2014.

Gaumond, M., Réthoré, P., and Bechmann, A.: Benchmarking of wind turbine wake models in large offshore wind farms, in: Proceedings of the Science of Making Torque From Wind, 9-11 October, Oldenburg, Germany, 2012.

Gaumond, M., Réthoré, P., Ott, S., Pena, A., Bechmann, A., and Hansen, K. S.: Evaluation of the wind direction uncertainty and its impact on wake modeling at the Horns Rev offshore wind farm, Wind Energy, 17, 1169-1178, doi:10.1002/we.1625, 2014.

Gebraad, P. M. O.: Data-Driven Wind Plant Control, PhD Thesis, Delft University of Technology, 2014. 
IEC 61400-12-1: Power performance measurements of electricity producing wind turbines, IEC, Geneva, Switzerland, 2005.

IEC 61400-12-2: Power performance of electricity-producing wind turbines based on nacelle anemometry, IEC, Geneva, Switzerland, 2013.

IEC TC88 WG6: Wind farm power performance testing working group draft, IEC, Geneva, Switzerland, 2005.

IEC TS 61400-26-1: Time-based availability for wind turbine generating systems, IEC, Geneva, Switzerland, 2011.

IEC TS 61400-26-2: Wind turbines - Part 26-2: Production-based availability for wind turbines, IEC, Geneva, Switzerland, 2014.

JCGM: Evaluation of measurement data - Guide to the expression of uncertainty in measurement, 1st Edn., International Organization for Standardization, Geneva, Switzerland, 2008.

$\mathrm{Ku}, \mathrm{H} .:$ Notes on the use of propagation of error formulas, J. Res. Natl. Bur. Stand., 70C, 263-273, 1966.

Marathe, N., Swift, A., Hirth, B., Walker, R., and Schroeder, J.: Characterizing power performance and wake of a wind turbine under yaw and blade pitch, Wind Energy, 19, 963-978, doi:10.1002/we.1875, 2016.

Mellinghoff, H.: Wind Farm Power Performance Verification, in: Proceedings of 8th German Wind Energy Conference, DEWEK, 22-23 November, Bremen, Germany, 2006.

Mittelmeier, N., Amelsberg, S., Blodau, T., Brand, A., Drueke, S., Kühn, M., Neumann, K., and Steinfeld, G.: Wind farm performance monitoring with advanced wake models, in: Proceedings of the European Wind Energy Association Offshore Conference, 19-21 November, Frankfurt, Germany, 2013.
Mittelmeier, N., Blodau, T., and Kühn, M.: Offshore wake model validation - Methodology for linking model results and operational data, in: Proceedings of 12th German Wind Energy Conference DEWEK, 19-20 May, Bremen, Germany, 2015.

Mortensen, N. G., Nielsen, M., and Jørgensen, H. E.: First Offshore Comparative Resource and Energy Yield Assessment Procedures (CREYAP), in: Proceedings of the European Wind Energy Conference, 19-21 November, Frankfurt, Germany, 2013.

Ott, S. and Nielsen, M.: Developments of the offshore wind turbine wake model Fuga, E-0046 Report 2014, DTU Wind Energy, Lyngby, Denmark, 2014.

Ott, S., Berg, J., and Nielsen, M.: Linearised CFD Models for Wakes, Ris $\emptyset$ National Laboratory, Roskilde, Denmark, 2011.

R_Core_Team: R: A Language and Environment for Statistical Computing, R Version 3.3.1, available at: https://www.r-project. org/ (last access: 21 June 2016), 2015.

Réthoré, P., Hansen, K., and Barthelmie, R.: Benchmarking of wind farm scale wake models in the EERA-DTOC project, in: International Conference on Aerodynamics of Offshore Wind Energy Systems and Wakes, 17-19 June, Lyngby, Denmark, 2013.

Steinfeld, G., Mittelmeier, N., Drüke, S., Brand, A., Hait, T., and Amelsberg, S.: Report on the results of benchmarks for wake models, ClusterDesign, Project No. 283145 (FP7 Energy), 2015.

Vollmer, L., Steinfeld, G., Heinemann, D., and Kühn, M.: Estimating the wake deflection downstream of a wind turbine in different atmospheric stabilities: an LES study, Wind Energ. Sci., 1, 129141, doi:10.5194/wes-1-129-2016, 2016. 